\title{
Pretreatment and Adsorption Effects on Stability and Morphology Structure of TCNQ-Based Porous Coordination
}

\author{
Moondra Zubir ${ }^{1}$, Marini Damanik ${ }^{2}$, HafniIndriati Nasution ${ }^{3}$ and MindaShafina Syafei ${ }^{4}$ \\ \{moondrazubir@unimed.ac.id\} \\ Chemistry Department, Faculty of Mathematics and Natural Science, State University of Medan, J1. \\ Willem Iskandar, Pasar V, Medan Estate, Medan, North Sumatera, Indonesia ${ }^{1,2,3,4}$
}

\begin{abstract}
Porous Coordination Polymers (PCPs) were synthesized using TCNQ and bipyridine anions that serve as connectors to form a 3-dimensional framework. In this study, zinc metal ions $\left(\mathrm{Zn}^{2+}\right)$ and manganese $\left(\mathrm{Mn}^{2+}\right)$ as the center of the complex compound coordination. XRD pattern of $\mathrm{Zn}$ (TCNQ-TCNQ) bpy.1.5Benzen 0T and 6T shows no difference, it suggest no magnetic field effect of $6 \mathrm{~T}$ on the formation of crystal structure. The magnetic field exhibits effect on the morphology of the crystals. The SEM image shows that the 6T crystal surface smoother and the particle size larger than the 0T crystal for both complex compounds. The XRD pattern shows the stability properties of the structure for both types of these complex compounds and the SEM image also shows the stability properties of the compounds after the pretreatment process and after the adsorption process..
\end{abstract}

Keywords: Magnetic fields, crystal structure, morphology, adsorption.

\section{Introduction}

Porous coordination polymers (PCPs) compounds have expanded beyond the scope of two other classes of porous material as inorganic material and carbon-based material. The crystal structure of PCPs become a new group of nano-porous crystals. (Noro, 2009 and Kitagawa, 2009). It's get high attention in porous material research because of their high surface area and large volume of free cages instead their interesting applications including gas purification, gas separation and catalysis (Seo and Matsudsa, 2009).

Porous polymer coordination was built on the self-design of metal ions and organic ligands and formed as crystalline materials which high flexibility obtained from the building of the skeleton through coordination covalent bonds (Ghosh and Kitagawa, 2009). The formation of structures and pore properties is very easy because the components of the preparation are selected. Using these properties, it's also possible to modify the pore surface with organic ligands that interact with certain molecules (Tanaka, 2008 and Sakamoto, 2009)

In this study, the TCNQ-based PCPs series of $\{[\mathrm{M}(\mathrm{TCNQ}-\mathrm{TCNQ}) \mathrm{bpy}] . x$. Benzene $\} \mathrm{n}$ was prepared using zinc $(\mathrm{Zn})$ and manganese $(\mathrm{Mn})$ as metal ions which have different magnetic properties as diamagnetic and paramagnetic respectively. TCNQ was used as ligand because it 
well known as an active redox ligand that can function as a good acceptor and weak or strong electron donors in valence numbers respectively are 0 . -1 or -2 (Shimomura and Kitagawa, 2006).Benzene was used as a solvent and many solvents have been used not only as a intermediate reaction, but also for topology arrangement. In micro-pore polymer coordination compounds, solvent molecules are extensively presence in the microporous, better known as "guest molecules" (Jalbout, 2008). Large diameter spaces are sufficient to accommodate benzene molecules because of the compatibility of cavity size and thickness (Shimomura and Kitagawa, 2007).

Magnetic fields brought a serious influence on their structure and properties. Magnetic field energy can provide the same direction for homogeneous of crystal formation. With the change in the formation of crystals due to magnetic fields could affected the physical properties and chemical properties in interacting with other molecules (Zubir and Ozeki, 2016).Furthermore, we synthesized both of PCPs series under 6T magnetic field and also without applied magnetic field (0T).

\section{Material and method}

In this research we used7,7,8,8-tetracyano-p-quinodimetana (TCNQ),4,4'-Bipiridin (bpy), Lithium Iodide (LI), and Acetonitrile, Diethyl Ether, Benzene and Methanol as solvents. Manganese (II) Nitrate Hexahydrate and Zinc (II) Nitrate Hexahydrate was used as metal ion in PCPs synthesis.

The Superconducting magnet 6T-Oxford Instrument IPS 125-9 with Helium compressor unit-Sumitomo CSW-71, were used for synthesis under 6T magnetic field. Crystal characterization and morphology were analyzed by using X-Ray Difraction (XRD) RigakuMultiflex with $\mathrm{Cu}-\mathrm{K} \alpha$ and Scanning Electron Microscopy - SEM JEOL JSM-6000 F.

\subsection{PCPs synthesis procedure}

\subsubsection{LiTCNQ synthesis}

A boiling solution of $5.1 \mathrm{~g}(0.1 \mathrm{~mol})$ TCNQ in $500 \mathrm{ml}$ of acetonitrile was mixed with a boiling solution of $10 \mathrm{~g}(0.3 \mathrm{~mol})$ Lithium Iodide (LI) in $25 \mathrm{ml}$ of acetonitrile. The mixture stayed in 1 hour at room temperature. Purple crystals will separate from the solution of dark brown and while still warm, the purple crystal was filtered and then washed with acetonitrile until the washing color becomes bright green. Finally. it washed with a large amount of ether until the washing color becomes clear (Kitagawa and Matsuda, 2007).

\subsection{2 [M(TCNQ-TCNQ)bpy]1.5Benzene 0T Synthesis}

Gently added LiTCNQ solution ( $2 \mathrm{mmol}, 422 \mathrm{mg}$ ) and 4,4'-Bipyridine (1 mmol, $156 \mathrm{mg}$ ) in a mixture of methanol and benzene $(100 \mathrm{ml}, 1: 1)$ into solution $\mathrm{M}\left(\mathrm{NO}_{3}\right)_{2} \cdot 6 \mathrm{H}_{2} \mathrm{O}(1 \mathrm{mmol}$, $\mathrm{Zn}=297 \mathrm{mg}$ and $\mathrm{Mn}=287 \mathrm{mg})$ in a mixture of methanol and benzene $(100 \mathrm{ml}, 1: 1)$ at $298 \mathrm{~K}$ and in the atmosphere of the nitrogen gas. The green crystals formed are collected by filtering. 


\subsection{3 [M(TCNQ-TCNQ)bpy]1.5Benzene 6T Synthesis}

LiTCNQ solution ( $2 \mathrm{mmol}, 422 \mathrm{mg}$ ) and 4,4'-Bipyridine (1 mmol, $156 \mathrm{mg}$ ) in a mixture of methanol and benzene $(100 \mathrm{ml}, 1: 1)$ was slowly added into solution $\mathrm{M}\left(\mathrm{NO}_{3}\right)_{2} \cdot 6 \mathrm{H}_{2} \mathrm{O}(1$ $\mathrm{mmol}, \mathrm{Zn}=297 \mathrm{mg}$ and $\mathrm{Mn}=287 \mathrm{mg})$ in a mixture of methanol and benzene $(100 \mathrm{ml}, 1: 1)$ at $298 \mathrm{~K}$ in the atmosphere of a nitrogen gas and put in 6 Tesla magnetic field centre. The green crystals formed are collected by filtering

\subsection{Crystal characterization}

The crystals formed were characterized by X-Ray Diffraction (XRD) Rigaku Multi Flex with $\mathrm{Cu}-\mathrm{K} \alpha$ monochromators and morphology of crystals analyzed by Scanning Electron Microscopy (SEM) JEOL JSM-6000 F.

\subsection{Structural stability study after pretreatment and adsorption}

Before determining the adsorption isotherm, the benzene molecule was removed by heating (pretreatment) at $413 \mathrm{~K}$ for 10 hours and under low pressure. XRD and SEM image were determined to see the changes in pretreatment to structural and morphological stability. At the beginning of the adsorption isotherm, $100 \mathrm{mg}$ of the sample was preheated at $383 \mathrm{~K}, 1$ $\mathrm{mPa}$ for 2 hours as a pretreatment to remove the remain solvent. The adsorption isotherm of oxygen gas is determined at $77 \mathrm{~K}$ and $1 \mathrm{mPa}$. After Adsorption, also observed changes in stability and morphology of the sample.

\section{Result and discussion}

XRD patterns of $\mathrm{Zn}$ and $\mathrm{Mn}$ complexes shows as iso-structure of each other which only one peak at 13.9 degree of Mn(TCNQ-TCNQ)bpy.1.5 Benzene which is not seen in $\mathrm{Zn}$ (TCNQ-TCNQ)bpy.1.5 Benzene. Mn[(TCNQ-TCNQ)bpy]1.5 0T and 6T also shows no significant difference of XRD patterns but some intensity changed in the 5 peaks at 13.84 , $15.28,18.48,22.98$ and 22.94 degrees, indicate differences structure formed due to magnetic field influences as shown in figure 1.

This slightly changed of XRD pattern due to the paramagnetic properties of manganese. This difference in intensity was calculated as the intensity ratio where 2 peaks as increase intensity and 3 peaks as decrease intensity. Before determining the adsorption isotherm, benzene was released from the cage by heating $413 \mathrm{~K}$ for 10 hours under low pressure. $\mathrm{Zn}[(\mathrm{TCNQ}-\mathrm{TCNQ})$ bpy]1.5 Benzene of 0T and 6T after pretreatment showed changed of crystals color to be dark green but Mn [(TCNQ-TCNQ) bpy] 1.5. 0T and 6T benzene did not changed the color after pretreatment.

Pretreatment for removing benzene content shows XRD patterns changed for all complex compounds. The four compounds release decomposition and decreased intensity compared to before treatment as shown in figures 1 and 2. This XRD pattern shows that these compounds are stable which are included into third generation compound of PCPs. XRD pattern of Zn [(TCNQ-TCNQ) bpy] 1.5. Benzene 0T and 6T after pretreatment showed no significant difference but in the other hand, Mn [(TCNQ-TCNQ) bpy] 1.5.Benzene 0T and 6T showed slightly differences in intensity of several peaks at 12.78, 17.34 and 17.44 degrees as decrease 
intensity while at 15.3 degrees as increase in intensity. The XRD patterns after adsorption for all complexes compounds $\mathrm{Zn}$ and $\mathrm{Mn}$ as shown similar XRD pattern comparison indicate these complexes compound was quite stable after adsorption process.
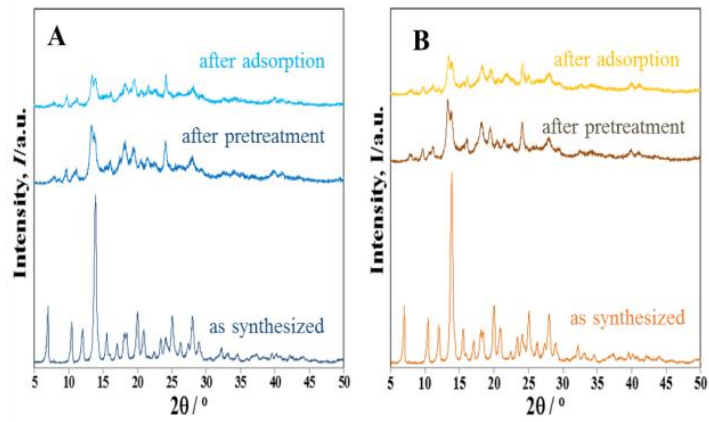

Fig.1. XRD Patterns of PCPs Zn[(TCNQ-TCNQ)bpy]1.5.Benzene of 0T (A) and 6T (B) in all treatment.
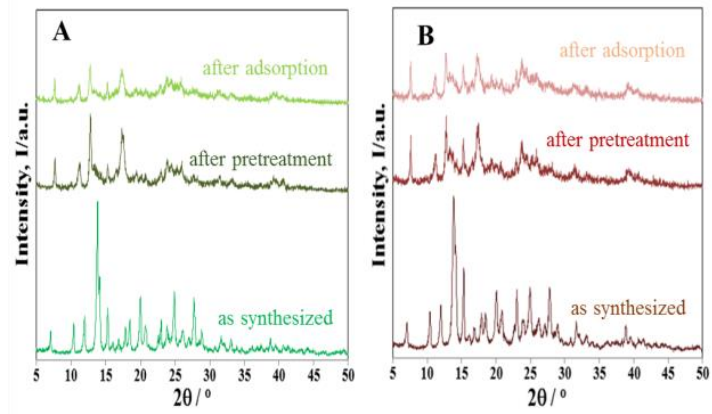

Fig.2. XRD Patterns of Mn[(TCNQ-TCNQ)bpy]1.5.Benzene of 0T (A) and 6T (B) in all treatment

SEM images were observed that there were no morphological differences between the crystals before and after the treatment as shown in Figures 3 and 4, indicate the pretreatment process to remove benzene from the frameworks were not affected to crystal morphology. SEM images after adsorption was compared with morphology after pretreatment and as synthesis crystal. 


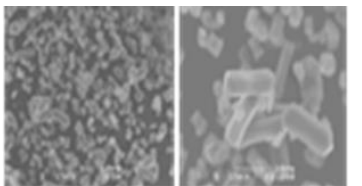

as synthesized

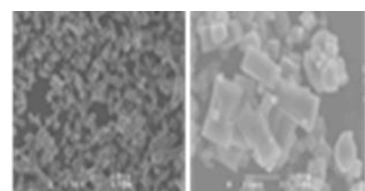

after pretreatment

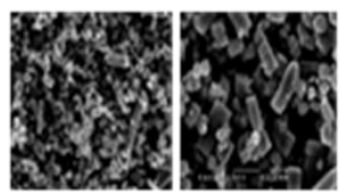

after adsorption

A

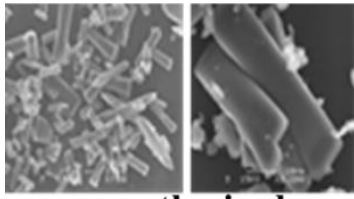

as synthesized

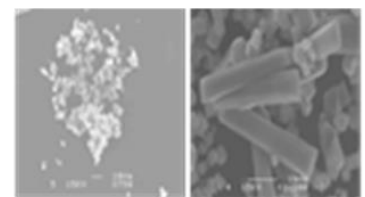

after pretreatment

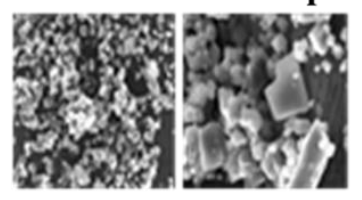

after adsorption

B

Fig.3. SEM images of PCPs Zn[(TCNQ-TCNQ)bpy]1.5.Benzene 0T (A) and 6T (B) in all treatment.

There were no significant morphological change after adsorption but the particles after adsorption became irregular due to the absorption of guest molecules but did not change the crystal structure. All morphologies of $\mathrm{Zn}$ complex compounds for comparison of all conditions are shown in figure 3 and for complex compounds $\mathrm{Mn}$ in figure 4. SEM images observed particle sizes of $\mathrm{Mn}$ complexes is bigger than $\mathrm{Zn}$ complexes due to magnetic properties induce strong interaction between metal ion and ligand. 


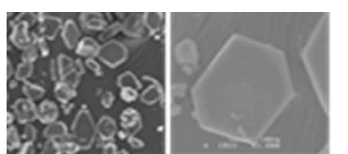

as synthesized
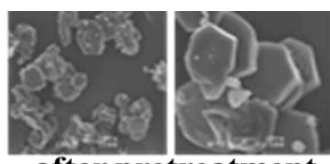

after pretreatment

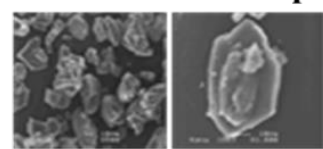

after adsorption

A

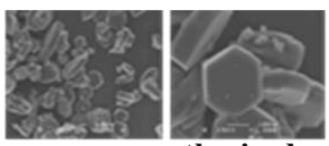

as synthesized

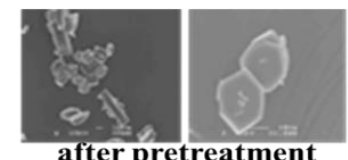

after pretreatment
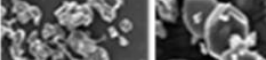

after adsorption

B

Fig.4. SEM images of $\mathrm{Zn}[(\mathrm{TCNQ}-\mathrm{TCNQ}) \mathrm{bpy}] 1.5$. Benzene $0 \mathrm{~T}(\mathrm{~A})$ and $6 \mathrm{~T}(\mathrm{~B})$ in all treatment.

The influence of magnetic fields in synthesis of Zn (TCNQ-TCNQ) bpy 1 1.5Benzene 0T and 6T crystals did not show differences in XRD patterns. Otherwise, SEM images, it shows that the shape is hexagonal at $0 \mathrm{~T}$, while the compound synthesized in the 6T magnetic field is rectangular and the particle size in the $\mathrm{Zn} 6 \mathrm{~T}$ compound is greater than that of the $\mathrm{Zn}$ 0T compound. Whereas for complex compounds Mn was slight changes to the XRD pattern but did not show significant differences for the morphology. The XRD pattern shows the stability of the structure for both types of complex compounds and SEM images also show the stability of the compound after the pretreatment process and after the adsorption process.

\section{References}

[1] Abraham F. Jalbout, Xin-Hua Li, Mohammad R. Hassan, G.M. GolzanHossain.:. Transition Metal Chemicals 33, 597 - 603 (2008)

[2] Daisuke Tanaka and Susumu Kitagawa.: Chem. Mater, 20, 922 - 931 (2008)

[3] Hirotoshi Sakamoto, Ryotaro Matsuda, SareeyaBureekaew, Daisuke Tanaka and Susumu Kitagawa.: Chemicals European Journal, 15, 4985 (2009)

[4] JoobeomSeo, Ryotaro Matsuda, Hirotoshi Sakamoto, Charlotte Bonneau and Susumu Kitagawa.:.Journal of American Chemical Society,131, 12792 - 12800 (2009)

[5] Satoru Shimomura, Ryotaro Matsuda, Takashi Tsujino, Takashi Kawamura and Susumu Kitagawa.: Journal of American Chemical Society 125, 1152 - 1153 (2006)

[6] Satoru Shimomura, Satoshi Horike, Ryotaro Matsuda and Susumu Kitagawa.:. Journal of American Chemical Society 129, 1990 (2007)

[7] Shin-ichiroNoro, Susumu Kitagawa, TomoyukiAkutagawa, Takayoshi Nakamur.: Journal of Polymer Science, 34, 240 - 279 (2009)

[8] Sujit K. Ghosh, SareeyaBureekaew and Susumu Kitagawa...Angew. Chem. Int. Ed., 47, 3403 (2008) 
[9] Susumu Kitagawa, Ryotaro Matsuda.:.Coordination Chemistry Reviews, 2512490 - 2509 (2007)

[10] Zubir, M., Hamasaki, A., Iiyama, T., Ohta, A., Ohki, H., Ozeki, S.:. Chem. Lett., 45, 362-364 (2016) 